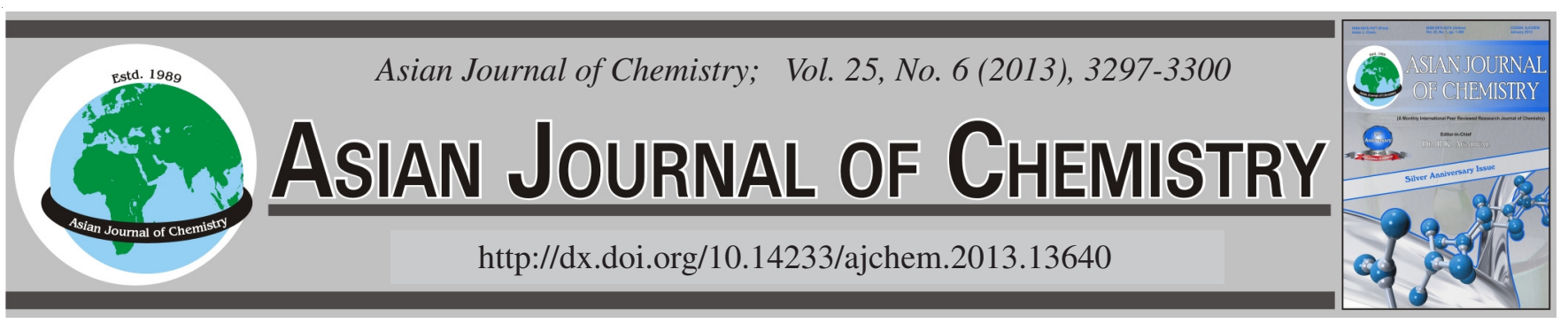

\title{
Organic Function Layer Interface Behaviour on Phosphorescent Material Device Performance
}

\author{
Zhixiang GaO ${ }^{1,2,3}$, YUying HaO ${ }^{1,3,4, *}$, Hua WANG ${ }^{1,3}$, Yanqin MiaO $^{1,3}$ and Bingshe XU ${ }^{1,3, *}$
}

${ }^{1}$ Key Laboratory of Interface Science and Engineering in Advanced Materials, Ministry of Education, Taiyuan University of Technology, 79 Yingze Street, Taiyuan 030024, Shanxi Province, P.R. China

${ }^{2}$ School of Physical Science and Electronics, Shanxi Datong University, Datong 037009, P.R. China

${ }^{3}$ Research Center of Advanced Materials Science and Technology, Taiyuan University of Technology, Taiyuan 030024, P.R. China

${ }^{4}$ Department of Physics and Optoelectronics, Taiyuan University of Technology, Taiyuan 030024, P.R. China

*Corresponding authors: E-mail: haoyuying@163.com; zhixianggao@163.com

(Received: 21 February 2012;

Accepted: 17 December 2012)

AJC-12571

\begin{abstract}
The interface charges influences on device performances which is captured in organic electroluminescent device (OLED) have become the hotspot of research in organic electroluminescent device, current conduction as well as the carrier and the electric field distribution is very crucial to the understanding of organic light emitting devices, (OLED) microscopic physical processes. This paper prepared a concave and convex interface structure of ITO/NPB/CBP $+\operatorname{Ir}(\mathrm{ppy})_{3} / \mathrm{liF} / \mathrm{Al}$ organic electrophosphorescent devices, with 4,4'-N,N'-two carbazole biphenyl (CBP) as the host material, Ir[ppy) ${ }_{3}($ tris(2-phenyl pyridine)iridium] as phosphorescent dopant, NPB as a hole transport layer. The different concave and convex emitting layer phosphorescent device of NPB/CBP $+\operatorname{Ir}(\mathrm{ppy})_{3}$ interface is studied and use the current density-voltage-brightness curve and current efficiency-voltage curve analysis device interface charge trapping and injected charge dynamics, determine the optimal convex number of light emitting layer, when the convex number is 3 , the maximum current efficiency of this device is $23 \mathrm{~cd} / \mathrm{A}$ and was $30 \%$ higher than traditional structure device.
\end{abstract}

Key Words: Organic light emitting devices, Interface, Phosphorescent, Luminous efficiency.

\section{INTRODUCTION}

Organic light emitting device (OLED) become research hotspot of modern flat-panel displays because of its low threshold voltage, high brightness, without backlight and self-luminous, wide viewing angle, etc. ${ }^{1}$. However, luminous efficiency of OLED is relatively low. In particular, issues such as device lifetime is shorter. This has become a technology bottleneck of its application. Up to now, improving the luminous efficiency and extending device lifetime OLED become a key research field of study ${ }^{2-4}$. The results show, performance of OLED depends on the performance of each functional layer. Therefore, improving the OLED material interface in the physical and chemical properties help to improve the luminous efficiency and extend the device lifetime ${ }^{5}$.

Interfaces of materials used in each functional layer, as well as interfaces between the functional layers, they assume the role of material transfer, such as the atom, electron, hole and exciton transfer, as well as energy transfer and have a direct impact on the luminous efficiency of OLED and device lifetime ${ }^{6}$. There are a large number of grain boundaries in each functional layer among OLED, as formless thin films (amorphous thin films), among that. That the carriers tend to clump in the grain boundary region, impedes on the one hand carrier injection and on the other hand, causes the grain boundary region to the non-radiative recombination centers, leading to OLED luminous efficiency lower ${ }^{7-9}$.

Recently, many research institutions have received some research achievements in the field of material interfaces of OLED. If a few nanometers thick layer of alkali metal halides (such as lithium fluoride, sodium fluoride, etc.) thin film is added between the electron injection layer and the metal electrode, OLED interface electron injection barrier can be effectively reduced, luminous efficiency is increased ${ }^{10,11}$. A doped layer of a few nanometers thick is added between the carrier transport layer and the light-emitting layer, containing both sides of the material. It on the one hand enhances the adhesion between layers, on the other hand can effectively avoid the accumulation of carriers in the layer interface region, helping to improve the luminous efficiency and extend the device lifetime ${ }^{12,13}$. Adding a few nanometers thick layer of metal oxide thin film between the anode hole injection layer, it has a positive effect on improving the hole injection efficiency and the luminous efficiency ${ }^{14}$.

In the present paper, we prepared a series of new structures the OLED device with different materials interface. By 
comparison, using this new structure will not only improve the luminous efficiency of OLED, but also to some extent, to avoid devices phosphorescence quenching and aging phenomenon under the high current density. Moreover, we studied the structure of a typical OLED, modified the interface of the typical structure, constructed inter-inserting structure in the organic/organic interface, studied photoelectric properties of the device by changing the inter-inserting number and analyzed the mechanism of this phenomenon.

\section{EXPERIMENTAL}

According to the experimental needs, the author designed and produced the corresponding films, fabricated with the concave-convex structure OLEDs (Fig. 1). Structure of the OLEDs is ITO/NPB $(x+m) / C B P: \operatorname{Ir}(p p y)_{3}(6 \%, 30 \mathrm{~nm}) / \mathrm{LiF}$ $(1 \mathrm{~nm}) \mathrm{Al}(100 \mathrm{~nm})(\mathrm{x}=25 \mathrm{~nm}, \mathrm{~m}=5 \mathrm{~nm}), " \mathrm{x}$ " is straight part of the thickness of the NPB. "m" is bulge part of the thickness of the NPB. The width of the bulge part is $0.5 \mathrm{~mm}$.

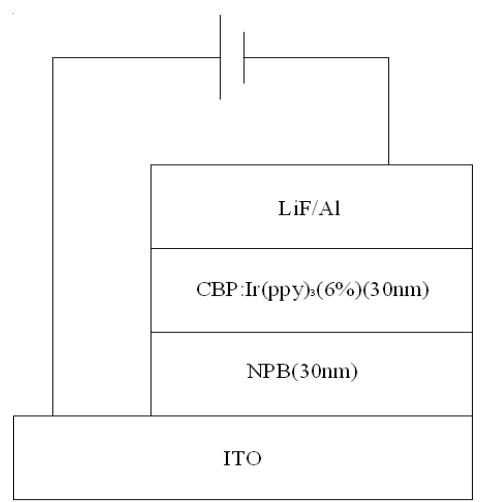

(D)

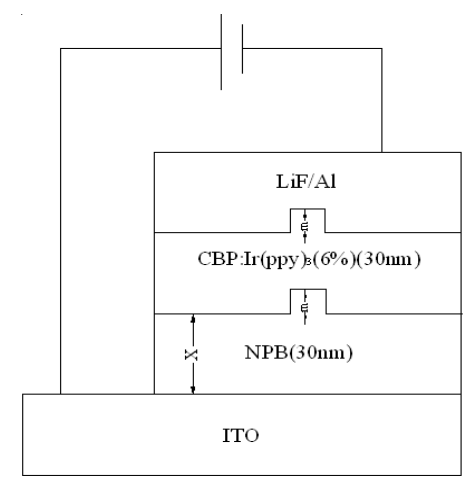

(D1)

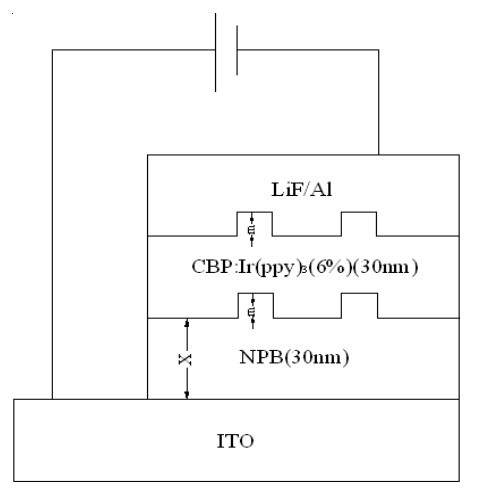

(D2)

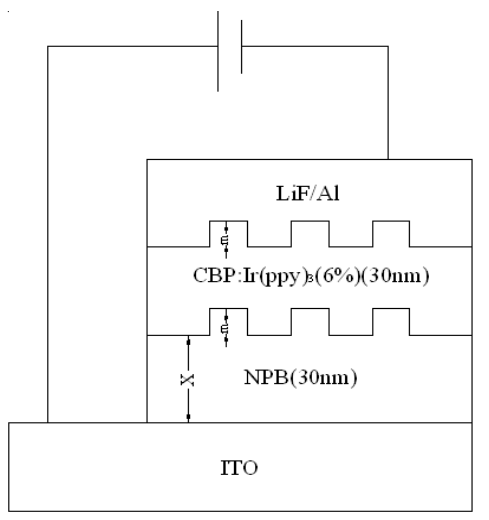

(D3)

Fig. 1. Schematic diagram of the devices (D) the traditional structure (D1) (D2) (D3) the inter-inserting interface structure

The chemical structures of organic materials(NPB, CBP, $\operatorname{Ir}(\text { ppy) })_{3}$ (Nichem Fine Technology Co.Ltd.) is shown in Fig. 2. The ITO-coated glass substrates (Shenzhen, China) with a sheet resistance of about $10 \Omega /$ sq were used as anodes. A series of OLEDs were fabricated. The OLEDs were made by following process. First, ITO-coated glasses were cleaned successively using deionized water, alcohol and acetone in an ultrasonic bath, dried in drying cabinet. Second, the films of N,N'-bis(1naphthyl)-N,N'-diphenyl-1,1'- biphenyl-4,4'-diamine (NPB), CBP:Ir(ppy $)_{3}$ and LiF were in turn grown on ITO-coated glass by thermal evaporation under high vacuum of $5 \times 10^{-4} \mathrm{~Pa}$ and the growth rates were all $0.1-0.2 \mathrm{~nm} / \mathrm{s}$ for organic materials and $0.05 \mathrm{~nm} / \mathrm{s}$ for LiF. Finally, Al metal as cathode was evaporated on $\mathrm{LiF}$ film at the rate of $1.0-1.5 \mathrm{~nm} / \mathrm{s}$. The thickness of the films was controlled by aquartz thickness monitor. The current density-voltage-luminance (J-V-L) and EL spectra of the OLEDs were measured simultaneously using a Keithley 2400 Source Meter and SpectraScan PR655 (Photo Research Inc.). The lifetime was measured using OEL-life11.10 (Beijing Ao Bodi Optical Technology Co., Ltd.). All the measurements were done at room temperature under ambient conditions without encapsulation except the lifetime.<smiles>[Pb]N(c1ccccc1)c1ccc(-c2ccc(N(c3ccccc3)c3cccc4ccccc34)cc2)cc1</smiles><smiles>c1ccc2c(c1)c1ccccc1n2-c1ccc(-c2ccc(-n3c4ccccc4c4ccccc43)cc2)cc1</smiles> 


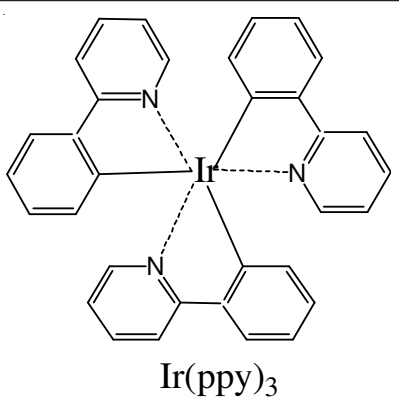

Fig. 2. Chemical structure of materials

\section{RESULTS AND DISCUSSION}

Fig. 3 showed that under the same drive voltage, the current density of the device with of the inter-inserting structure is significantly lower than device with a traditional structure. When the voltage is greater than $9 \mathrm{~V}$, there are nearly the same current densities for device D1 and device D2, as the lowest devices and the lower one for device D3. When the voltage is less than $9 \mathrm{~V}$, the current densities of the device D1 and D2 are basically the same and the lowest. As, under the conditions of the high current density, the $\mathrm{C}-\mathrm{N}$ bonds outside CBP molecules in the excited state homolysis, the formation of by-products generated by phosphorescence quenching center. When the projection number of the device structure is optimum, to a certain extent, reduce the device's current density, which effectively inhibit the formation of phosphorescence quenching centers and ultimately improve the brightness of device and extend the lifetime of device. In addition, it helps to reduce triplet-triplet quenching occurs under the high current density, to improve the stability of device. The maximum current efficiency of the device is basically just lighting and the low current density, phosphorescence is easy to quench under high current density.

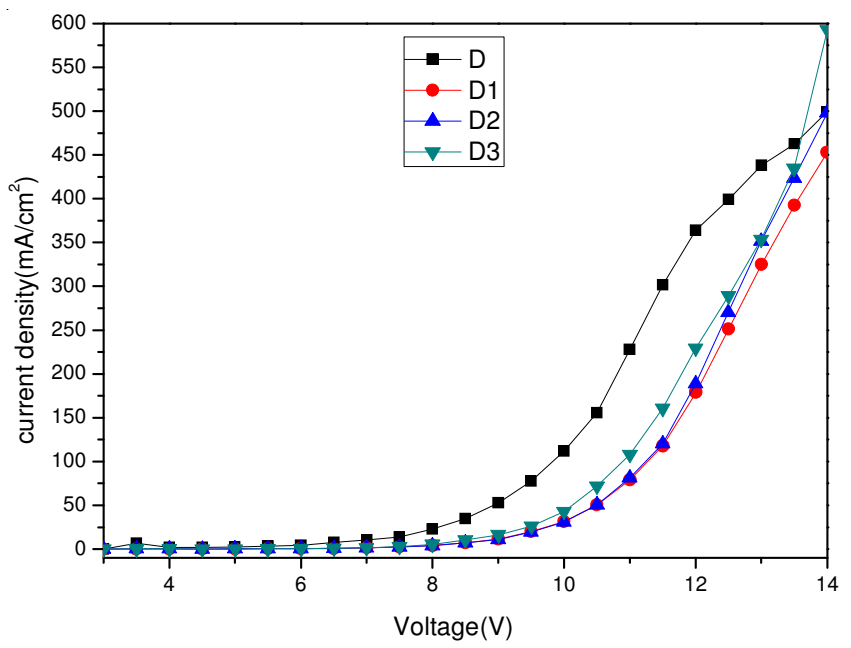

Fig. 3. J-V curves of devices

The L-V curves of devices $\mathrm{D}_{-} \mathrm{D}_{3}$ are shown in Fig. 4. From the Fig. 4, we can see that the device D3, compared to the other device, only requires a minimum voltage to be bright and has the highest brightness. Devices D1, D2 and D can basically be light in the same time. But when the voltage is greater than $12 \mathrm{~V}$, the luminance of $\mathrm{D}$ begins to decrease, but the devices of D1 and D2 reaches a maximum brightness in

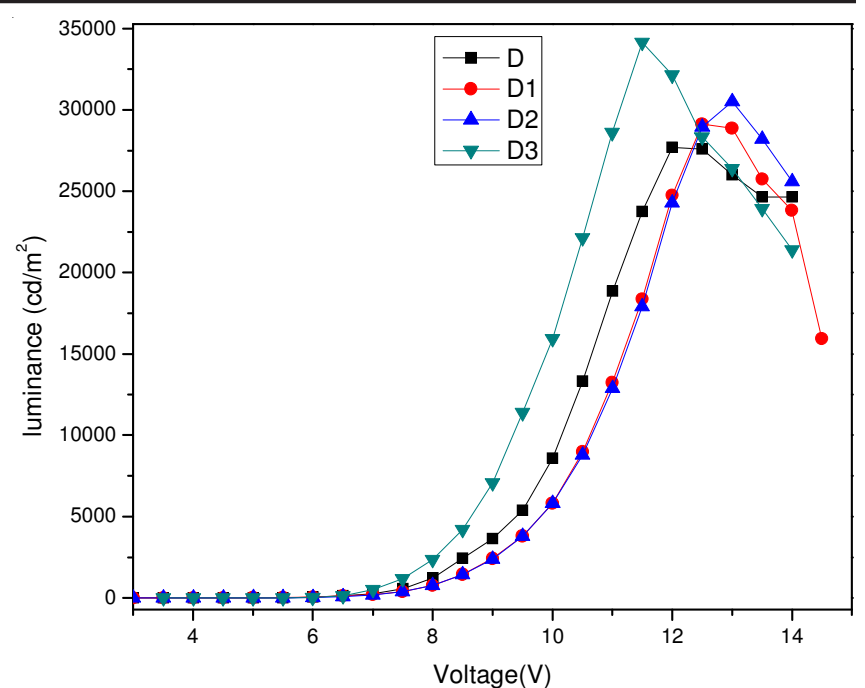

Fig. 4. L-V curves of devices

succession. Because the electronic through the low level transmission and the hole through NPB transmission firstly form the exciton in the light-emitting layer interface, the relative interface of device D3 has larger contact area. Furthermore, the electric field in low electrical level is strong and the ability to transport carriers also is strong, so the device D3 turns light at first.

The $\eta$ A-J curves of devices D-D3 are shown in Fig. 5. Current efficiency of devices D3, D2, D1 and D was 23, 22.6, 21.7 and $17.7 \mathrm{~cd} / \mathrm{A}$, respectively. Compared with device $\mathrm{D}$, the current efficiency were increased by 30,29 and $26 \%$, respectively. With the increase in the number of square wave cycle, as composite interface is bigger, the device current efficiency gets higher and higher. The inter-inserting interface structure can increase the electron transmission efficiency, disperse electronic accumulated in the interface, transmit mass electronic so as to reach equilibrium with the hole from the hole transport layer NPB and composite into excitons in the luminous interface. Therefore, it can improve current efficiency of the device. Current efficiency improvement of the concaveconvex structure of devices may be attributed to the following reasons: First, the inter-inserting structure is equivalent to the entire device is divided into NPB thickness of 25, 30 and 25 $\mathrm{nm}$ of three parallel devices. Current density of the parallel connection structure of device is lower than the NPB thickness of $30 \mathrm{~nm}$ device D. Second, inter-inserting structure can increase the carrier recombination interface area, disperse NPB/CBP: $\operatorname{Ir}(\text { ppy })_{3}$ interface triplet exciton, reduce the triplet exciton quench chance and improve the triplet exciton radiative decay chance. Thirdly, the inter-inserting structure will be helpful to couple some of the light existing inside the device in the form of the optical waveguide to outside the device. Because the device D has not inter-inserting adjacent NPB hole transmission layer, leading to its current efficiency is lower than the device D1, D2 and D3.

\section{Conclusion}

In the present paper, we changed the traditional organic electroluminescent phosphorescent devices NPB/CBP: $\operatorname{Ir}(\mathrm{ppy})_{3}$ of the interface structure, produced a inter-inserting structure of the device. The results indicate that the device has a maximum 


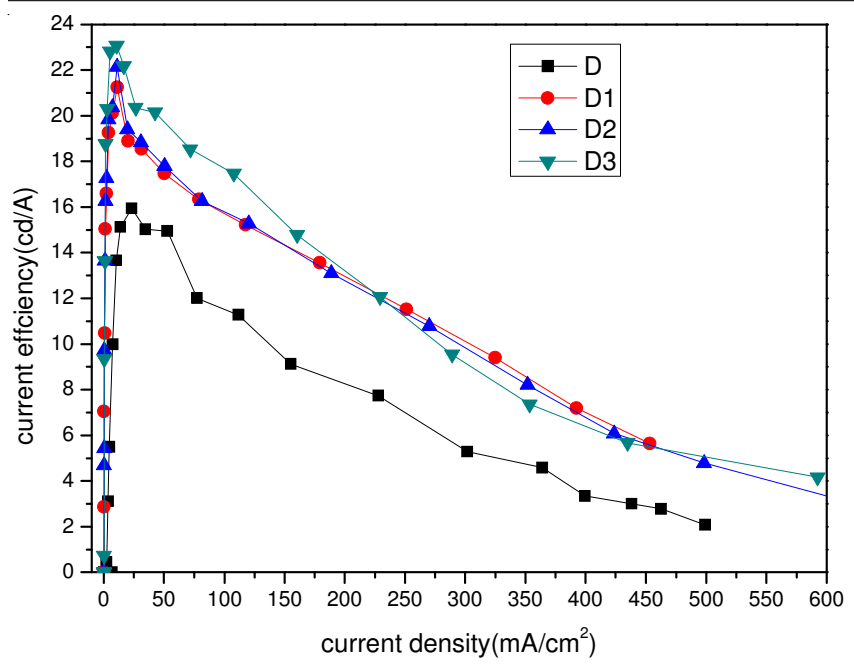

Fig. 5. ๆA-J curves of devices

current efficiency in the inter-inserting structure device when the inter-inserting number is 3 . The maximum current efficiency is $23 \mathrm{~cd} /$ A.Compared with the traditional structure of the device current efficiency increased by $30 \%$.

\section{ACKNOWLEDGEMENTS}

This research work was financially supported by National Natural Scientific Foundation of China (21071108,60976018),
Program for Changjiang Scholar and Innovative Research Team in University (IRT0972) and Shanxi Natural Scientific Foundation (2008011008, 2010021023-2).

\section{REFERENCES}

1. C.W. Tang and S.A. Vanslyke, Appl. Phys. Lett., 51,13 (1987)

2. M. H. Song, D. Kabra, B. Wenger, R.H. Friend and H.J. Snaith, Adv. Funct. Mater, 19, 2130 (2009).

3. W.-Y. Dan, Y.-Y. Di, D.-M. Zhang, D.-H. He and Y.-P. Liu, Asian J. Chem., 23, 1015 (2011).

4. L.L. Han, D.F. Yang, W.L. Li, B. Chu, Y.R. Chen, Z.S. Su, D.Y. Zhang, F. Yan, S.H. Wu, J.B. Wang, Z.Z. Hu and Z.Q. Zhang, Appl. Phys. Lett., 94, 163303 (2009).

5. B. Dandrade, Nat. Photonics, 1, 33 (2007).

6. Y.Y. Hao, J.F. Lei and B.S. Xu et al., Curr. Appl. Phys. (2009).

7. D.E. Loy, B.E. Koene and M.E. Thompson, Adv. Funct. Mater, 12, 245 (2002).

8. R. Sebastian, L. Frank, G. Schwartz, N. Seidler, K. Walzer, B. Lüssem and K. Leo, Nature, 459, 234 (2009).

9. F. Papadimitrakopoulos, X.M. Zhang, D.L. Thomsen and and K.A. Higginson, Chem. Mater, 8, 1363 (1996).

10. D.Q. Zhang, Y. Li, G.H. Zhang, Y.D. Gao, L. Duan, L.D. Wang and Y. Qiu, Appl. Phys. Lett., 92, 073301 (2008).

11. J. Lee, Y. Park, D. Y. Kim, H. Y. Chu, H. Lee and L.-M. Do, Appl. Phys. Lett., 82, 173 (2003).

12. L.S. Liao, W.K. Slusarek, T.K. Hatwar, M.L. Ricks and D.L. Comfort, Adv. Mater., 20, 324 (2008).

13. M.Y. Chan, S.L. Lai, K.M. Lau, M.K. Fung, C.S. Lee and S.T. Lee, Adv. Funct. Mater. 17, 2509 (2007)

14. F. Wang, X. Qiao, T. Xiong and D. Ma,Org. Electronics, 9, 985 (2008). 